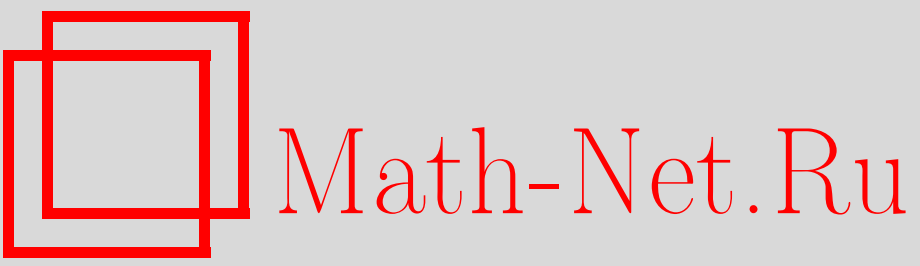

А. В. Селиванов, Существование и единственность мартингальных мер в экспоненциальных моделях Леви, УМН, 2004, том 59, выпуск 3, 179-180

DOI: https://doi.org/10.4213/rm753

Использование Общероссийского математического портала Math-Net.Ru подразумевает, что вы прочитали и согласны с пользовательским соглашением

http://www.mathnet.ru/rus/agreement

Параметры загрузки:

IP : 35.174 .16 .151

26 апреля 2023 г., 13:32:27 


\title{
СУЩЕСТВОВАНИЕ И ЕДИНСТВЕННОСТЬ МАРТИНГАЛЬНЫХ МЕР В ЭКСПОНЕНЦИАЛЬНЫХ МОДЕЛЯХ ЛЕВИ
}

\author{
А. В. СЕливАнов
}

В финансовой математике важную роль играет вопрос существования и единственности мартингальной меры в различных моделях цен. Это вопрос о наличии арбитража и полноте в соответствующей модели рынка (см. [1; гл. V, VII]).

В данной работе мы даем ответ на этот вопрос для двух моделей цен, популярных в современной финансовой математике.

1. Экспоненциальная модель Леви, т.е. модель, в которой процесс дисконтированной цены имеет вид

$$
S_{t}=e^{X_{t}},
$$

где $X$ - процесс Леви.

2. Экспоненциальная модель Леви с заменой времени, т.е. модель, в которой процесс дисконтированной цены имеет вид

$$
S_{t}=e^{(X \circ \tau) t}
$$

где $X$ - процесс Леви, $\tau$ - не зависящий от $X$ неубывающий процесс, $(X \circ \tau){ }_{t}=X_{\tau_{t}}$.

Мы рассмотрим случаи как конечного, так и бесконечного временного горизонта. Предположим, что процессы заданы на вероятностном пространстве $(\Omega, \mathscr{F}, \mathrm{P})$. Рассмотрим фильтрацию $\mathscr{F}_{t}=\sigma\left(S_{u}, u \leqslant t\right), t \geqslant 0$.

Классы мартингальных мер, которые мы будем изучать, - это классы, возникающие в фундаментальной теореме теории арбитража (ФТТА). Для случая непрерьвного времени эта теорема может быть сформулирована разными способами. Предположим, что процесс дисконтированной цень $S$ является семимартингалом.

В традиционной формулировке $\Phi$ ТТА, полученной $\Phi$. Делбаеном и В. Шахермайером в [2], утверждается, что в модели $\left(\Omega, \mathscr{F},(\mathscr{F} t)_{t \in[0, T]}, \mathrm{P},\left(S_{t}\right)_{t \in[0, T]}\right)$ отсутствует бесплатный ленч c исчезающим риском тогда и только тогда, когда $\mathscr{M}_{\sigma} \neq \varnothing$, где

$$
\left.\mathscr{M}_{\sigma}=\{\mathrm{Q} \sim \mathrm{P}: S \text { является (F्F } t, \mathrm{Q}) \text { - } \sigma \text {-мартингалом }\right\}
$$

(элемент из $\mathscr{M}_{\sigma}$ назьвается $\sigma$-мартингальной мерой). Этот же результат справедлив и для модели с бесконечным временным горизонтом.

Однако понятие арбитража может быть введено иначе, чем это делается в [2] (см. [1; гл. VII, $\S 2])$. В некоторых случаях (см., например, [3]) ФТТА для моделей с конечным временным горизонтом формулируется с использованием множества

$$
\mathscr{M}=\left\{\mathrm{Q} \sim \mathrm{P}: S \text { является }\left(\mathscr{F}_{t}, \mathrm{Q}\right) \text {-мартингалом }\right\}
$$

(элемент из $\mathscr{M}$ назьвается мартингальной мерой).

В силу некоторых соображений оказывается полезным рассматривать следующее множество для моделей с бесконечным временным горизонтом:

$$
\mathscr{U} M=\left\{\mathrm{Q} \sim \mathrm{P}: S \text { является }\left(\mathscr{F}_{t}, \mathrm{Q}\right) \text {-равномерно интегрируемым мартингалом }\right\}
$$

(элемент из $\mathscr{U} M$ назьвается равномерно интегрируемой мартингальной мерой).

Заметим, что множества $\mathscr{M}$ и $\mathscr{U} M$ могут быть рассмотрены вместо $\mathscr{M}_{\sigma}$ в традиционной ФТТА при некоторых дополнительных ограничениях на модель (см. [4]).

Перейдем к моделям (1) и (2). Предположим, что $X$ - это ненулевой процесс Леви с триплетом характеристик $(b, c, \nu)$ (относительно произвольно взятой функции усечения), $\tau$ - это отличньй от константы неубьвающий непрерьвньй справа и имеющий пределы слева процесс, не зависящий от $X$. Через $|A|$ мы обозначим число элементов в множестве $A$, через $\delta_{a}$ - дельта-меру, сосредоточенную в точке $a$. 
Теорема 1. Рассмотрим модель (1) с конечным временным горизонтом.

(i) Мь имеем $\mathscr{M}_{\sigma}=\varnothing \Leftrightarrow \mathscr{M}=\varnothing \Leftrightarrow X$ или $-X$ не убьвает.

(ii) Предположим, что $\mathscr{M}_{\sigma} \neq \varnothing$. Тогда

$$
\left|\mathscr{M}_{\sigma}\right|=1 \Leftrightarrow|\mathscr{M}|=1 \Leftrightarrow c \neq 0, \nu=0 \text { uли } c=0, \nu=\alpha \delta_{a} .
$$

ЗАмечАниЕ. Случай $c \neq 0, \nu=0$ отвечает модели Блэка-Шоулса, случай $c=0, \nu=\alpha \delta_{a}$ отвечает модели Мертона.

Теорема 2. Рассмотрим модель (2) с конечным временным горизонтом.

(i) Мы имеем $\mathscr{M}_{\sigma}=\varnothing \Leftrightarrow \mathscr{M}=\varnothing \Leftrightarrow X$ или - ие убьвает.

(ii) Предположим, что $\mathscr{M}_{\sigma} \neq \varnothing$. Тогда

$$
\left|\mathscr{M}_{\sigma}\right|=1 \Leftrightarrow|\mathscr{M}|=1 \Leftrightarrow \begin{aligned}
& \tau-\text { детерминированныц̆, непрерывный процесс } и \\
& c \neq 0, \nu=0 \text { или } c=0, \nu=\alpha \delta_{a} .
\end{aligned}
$$

Теорема 3. Рассмотрим модель (1) с бесконечным временным горизонтом.

(i) $М$ иь имеем

$$
\mathscr{M}_{\sigma} \neq \varnothing \Leftrightarrow \begin{aligned}
& S \text { является }\left(\mathscr{F}_{t}, \mathrm{P}\right) \text {-мартингалом или } \\
& \mathrm{E} S_{1}<1 \text { и скачки прочесса } X \text { не ограничень сверху. }
\end{aligned}
$$

(ii) Предположим, что $\mathscr{M}_{\sigma} \neq \varnothing$. Тогда $\left|\mathscr{M}_{\sigma}\right|=1 \Leftrightarrow c \neq 0, \nu=0$ или $c=0, \nu=$ $\alpha \delta_{a}$.

(iii) $M$ вь имеем $\mathscr{U} \mathscr{M}=\varnothing$.

Теорема 4. Рассмотрим модель (2) с бесконечным временным горизонтом. Предположим, ито $\tau_{\infty}=\infty$ P-n.н. Тогда $\mathscr{U} \mathscr{M}=\varnothing$.

ЗАмечАНИЕ. Мы видим, что структура множеств $\mathscr{M}_{\sigma}$ и $\mathscr{M}$ (в смысле существования и единственности элемента) одинакова для модели с конечным временным горизонтом. Структура же множеств $\mathscr{M}_{\sigma}$ и $\mathscr{U} \mathscr{M}$ оказывается различной для модели с бесконечным временным горизонтом.

Автор благодарен А. С. Черному за руководство этой работой и важные замечания.

\section{СПИСОК ЛИТЕРАТУРЫ}

[1] А.Н. Ширяев. Основы стохастической финансовой математики. Т. 2. М.: Фазис, 1998. [2] F. Delbaen, W. Schachermayer // Math. Ann. 1998. V. 312. № 2. P. 215-250. [3] C.A. Sin. Strictly local martingales and hedge ratios on stochastic volatility models // Ph. D. Dissertation. Ithaka, NY: Cornell University, Graduate School, 1996. [4] F. Delbaen, W. Schachermayer // Math. Ann. 1994. V. 300. № 3. P. 463-520. 